\title{
Limitação do suporte de vida pediátrico: argumentações éticas
}

Grace Kelly Paranhos ${ }^{1}$, Sergio Rego ${ }^{2}$

\section{Resumo}

Objetivos: Conhecer os argumentos éticos de médicos intensivistas pediátricos de uma unidade de terapia intensiva pediátrica da cidade do Rio de Janeiro para justificar suas decisões em casos de limitação de suporte de vida e analisá-los criticamente. Métodos: Trata-se de estudo de caso, descritivo. Foram realizadas 17 entrevistas semiestruturadas com intensivistas pediátricos de uma unidade de terapia intensiva pediátrica de alta complexidade do Rio de Janeiro. Resultados: Os entrevistados desconhecem a aplicação e as etapas do processo de limitação do suporte de vida, utilizando uma tendência paternalista nas tomadas de decisão. Conclusões: Medidas educativas relacionadas com o processo de morrer devem ser incentivadas durante a formação médica, desenvolvendo o diálogo e o reconhecimento do outro como agente moral. A bioética pode ajudar no desenvolvimento crítico desses profissionais, auxiliando na resolução dos conflitos éticos que surgem não só no processo de limitação do suporte de vida, como em outras áreas da medicina.

Palavras-chave: Bioética. Cuidados paliativos na terminalidade da vida. Pediatria. Unidades de terapia intensiva. Educação médica.

\section{Resumen}

\section{Limitación del soporte de vida pediátrico: argumentos éticos}

Objetivos: Conocer los argumentos éticos de médicos de cuidados intensivos pediátricos de una unidad de cuidados intensivos pediátrica de la ciudad de Río de Janeiro para justificar sus decisiones en los casos de limitación de soporte vital y analizarlos críticamente. Métodos: Se trata de un estudio de caso, descriptivo. Se realizaron 17 entrevistas semiestructuradas con los intensivistas pediátricos en una Unidad de Cuidados Intensivos Pediátricos de alta complejidad en la ciudad de Río de Janeiro. Resultados: Los médicos entrevistados desconocen la aplicación y las etapas del proceso de Limitación del Soporte de Vida, utilizando en su lugar una tendencia paternalista en la toma de decisiones. Conclusiones: Las actividades educativas relacionadas con el proceso de muerte deben ser incentivadas durante la formación médica, para facilitar el desarrollo del diálogo y el reconocimiento del otro como un agente moral. La Bioética puede ayudar al desarrollo crítico de estos profesionales, auxiliándolos en la resolución de los conflictos éticos que surjan, no solo en el proceso de Limitación del Soporte de Vida, como en otras áreas de la medicina.

Palabras-clave: Bioética. Cuidados paliativos al final de la vida. Pediatría. Unidades de cuidados intensivos. Educación médica.

\section{Abstract}

\section{Limitation of pediatric life support: ethical arguments}

Objectives: To know the ethical arguments of pediatric intensive care physicians in order to justify decisions in the field of limitation of life support, in a pediatric intensive care unit of the city of Rio de Janeiro, analyzing them critically. Methods: This is a descriptive case study. 17 semi-structured interviews were performed with intensive care physicians that worked in a high complexity pediatric intensive care unit in Rio de Janeiro. Results: The physicians adopted a paternalistic tendency in the decision making of limitation of life support, and the interviewed people were not aware of its application and the steps of the process. Conclusions: Educational activities related to dying process should be encouraged as the dialogue and recognition of others as a moral agent. Bioethics can be useful in this critical development helping the resolution of ethical conflicts not only in the limitation of life support process but also in other areas of medicine.

Keywords: Bioethics. Palliative care in terminally life. Pediatrics. Intensive care units. Medical education.

Aprovação CEP Ensp 82/10

1. Doutoranda paranhosgrace@gmail.com - Instituto Fernandes Figueira/Fundação Oswaldo Cruz (IFF/Fiocruz), Rio de Janeiro/RJ, Brasil 2. Doutor rego@ensp.fiocruz.br - Escola Nacional de Saúde Pública Sergio Arouca/Fundação Oswaldo Cruz (Ensp/Fiocruz), Rio de Janeiro/RJ, Brasil.

Correspondência

Grace Kelly Paranhos - Avenida Prefeito Dulcídeo Cardoso, 3.333/302 Barra da Tijuca CEP 22630-022. Rio de Janeiro/RJ, Brasil.

Declaram não haver conflito de interesse. 
Em um fenômeno social no qual o avanço no desenvolvimento das tecnologias no campo das ciências médicas teve um papel importante, a morte deslocou-se, na primeira metade do século XX, do ambiente familiar para o hospitalar. $O$ hospital passou a ser o local onde o cuidado médico pode ser oferecido e a morte passa a ser um fenômeno técnico causado pela parada dos cuidados, ou seja, de maneira mais ou menos declarada, por decisão do médico e da equipe hospitalar ${ }^{1}$.

As unidades de terapia intensiva (UTI) surgiram a partir da década de 1950, com a percepção de que era mais efetivo para o cuidado reunir em um único ambiente os pacientes que estavam utilizando ventilação mecânica ${ }^{2}$. Sendo o local do hospital em que os pacientes mais graves eram alocados e com mais recursos de alta tecnologia, a UTI passou a receber também pacientes crônicos, com a esperança de que o uso dessas tecnologias impedisse, ou ao menos retardasse de forma significativa, sua morte. Tal providência, por outro lado, aumentou também o sofrimento fútil de alguns enfermos, resultado do uso desmedido desse tipo de tecnologia.

A partir dos anos 1990, o cuidado na qualidade do fim de vida começa a receber mais atenção, e discussões sobre questões éticas envolvendo esse tema, como a obstinação terapêutica, passaram a ser muito frequentes no campo acadêmico, embora tais debates não tenham sido frequentes no meio acadêmico médico brasileiro ${ }^{3}$. 0 modelo biomédico de cuidado associado ao novo paradigma tecnológico impôs a alguns profissionais a obrigação de oferecer aos pacientes todo e qualquer recurso tecnocientífico para o enfrentamento das situações clínicas. Os verdadeiros benefícios dessas ações são muitas vezes postos em segundo plano, infligindo sofrimento e dor aos pacientes, sem que seja alcançado um resultado satisfatório em termos de cura ou controle de doença, o que é descrito como distanásia ${ }^{4,5}$.

Devemos ter clareza de que o grande desenvolvimento da tecnologia a serviço da medicina é um fenômeno relativamente recente, cujo início se dá em torno da década de 1960. Esse desenvolvimento tecnológico, seja na forma de novos medicamentos ou de equipamentos diagnósticos e terapêuticos, foi acompanhado do avanço de novas técnicas. Com isso, a capacidade de intervenção terapêutica em um paciente grave tornou-se incomensuravelmente maior, dificultando determinar o momento em que tecnicamente não há mais como ajudar um paciente, o que faz que a obstinação te- rapêutica e a distanásia sejam, cada vez mais, possibilidades concretas.

O processo de limitação do suporte de vida (LSV) se contrapõe à futilidade terapêutica, tentando evitar processos de morte dolorosos. Ele compreende a ordem de não reanimar (manutenção de todas as medidas curativas até que a morte ocorra por parada cardiorrespiratória), a ordem de retirada (suspensão de uma ou várias medidas que estejam prolongando o processo de morrer) e a ordem de não oferta (não iniciação de medidas de suporte curativo que possam prolongar o processo de morte de um indivíduo) ${ }^{6,7}$. Publicações recentes mostram o aumento do emprego da LSV em até $90 \%$ nos ambientes de terapia intensiva ${ }^{8}$. Isso também é uma realidade em estudos brasileiros, muito embora estes demonstrem, na verdade, que mais da metade das medidas de LSV correspondem apenas à ordem de não reanimar ${ }^{6}$. Os estudos em pediatria ainda não são muito frequentes, mas já revelam que, assim como na terapia intensiva adulta, até $72 \%$ dos óbitos em unidades de terapia intensiva pediátrica (Utip) foram precedidos de alguma medida de limitação ${ }^{9}$.

A bioética, que se preocupa em analisar os argumentos morais a favor e contra determinadas práticas humanas que afetam a qualidade de vida e o bem-estar dos humanos e em tomar decisões baseadas nas análises anteriores ${ }^{10}$, pode auxiliar os profissionais de saúde a lidar melhor com conflitos emergentes nos cuidados de fim de vida, facilitando as tomadas de decisão na medida em que as questões morais forem apropriadamente consideradas e a decisão seja bem fundamentada.

Considerando a escassez de trabalhos com este foco, elaboramos nossas hipóteses a partir da experiência prática dos autores. Consideramos que os médicos expressariam o conflito no momento decisório da LSV como a tensão entre o princípio da sacralidade da vida e o da autonomia do paciente e no uso inapropriado de recursos, levando-se em conta a escassez desses recursos nos sistemas de saúde. Cabe ressaltar que nossa compreensão do princípio da sacralidade da vida está em consonância com o expresso por Diniz, que o distingue do princípio da santidade da vida. A sacralidade é um princípio laico que assegura o valor moral da existência humana e fundamenta diferentes mecanismos sociais que garantem o direito de estar vivo. [...] Reconhecer o valor moral da existência humana não é o mesmo que supor sua intocabilidade. O princípio da santidade da vida é de fundamento dogmático e religioso ${ }^{11}$. 


\section{Método}

Este foi um estudo de caso, descritivo, em que foram tomadas como objeto de análise as falas de médicos intensivistas pediátricos, contratados por uma clínica privada da cidade do Rio de Janeiro para trabalhar em sua Utip, sobre a limitação de suporte de vida.

Não existem informações atualizadas disponíveis sobre o número de Utip ou mesmo sobre o número de postos de trabalho. O Instituto Brasileiro de Geografia e Estatística disponibiliza dados agregados por cidade, mas não distingue as unidades de terapia intensiva de adultos das pediátricas. A meIhor informação disponível para o Rio de Janeiro é a oferecida por Barbosa, com dados obtidos em visita a cada um dos estabelecimentos. Naquela ocasião foram identificadas na região metropolitana do estado apenas 11 unidades de terapia intensiva destinadas exclusivamente a pacientes pediátricos e 25 unidades mistas. $O$ número de leitos disponíveis no final do século passado era de 59 exclusivos para pacientes pediátricos e 223 mistos ${ }^{12}$.

Os médicos foram os sujeitos de estudo escoIhidos para esta pesquisa por representarem a figura central na decisão do processo de limitação do suporte de vida. Foi selecionada para este estudo uma unidade de terapia intensiva pediátrica de alta complexidade de uma clínica do setor privado da cidade do Rio de Janeiro. A escolha dessa clínica teve como objetivo assegurar uma possibilidade maior de ocorrências da prática de limitação do suporte de vida.

Como são poucas as unidades desse tipo na cidade do Rio de Janeiro e a maioria dos profissionais que atuam em Utip trabalha em mais de uma unidade, entendeu-se que essa unidade era adequada ao estudo de caso. Reforça a possibilidade de generalizar as informações, da mesma maneira, o fato de os processos de trabalho e a forma como o trabalho se organiza nas Utip não diferirem essencialmente de uma unidade para outra, sendo compostas tanto por médicos plantonistas quanto por médicos que exercem a função de rotina do serviço, que comparecem diariamente à unidade.

Na Utip estudada existiam 16 plantonistas com carga horária de 18 horas distribuídas em plantões de 12 horas semanais, sendo um plantonista no período diurno e dois plantonistas no noturno, com revezamento em plantões de 12 horas em finais de semanas alternados. O serviço contava também com três médicos diaristas que exerciam suas funções nos períodos da manhã e da tarde, de maneira que durante o dia existisse sempre na Utip um plantonista e pelo menos um médico da rotina. A situação de todos os pacientes, assim como a programação de exames e suas condutas de acordo com seu estado clínico e diagnóstico, era discutida entre plantonistas e diaristas em "rounds" cotidianos que contavam também com a participação da enfermeira de plantão, fisioterapeuta e psicóloga.

A pesquisadora principal deste estudo é um dos 19 intensivistas pediátricos que trabalhavam na Utip selecionada. Esse conhecimento prévio dos sujeitos de pesquisa pode ter dificultado o relato de práticas que fossem passíveis de ser consideradas ilegais ou antiéticas. A garantia de confidencialidade foi reforçada com o intuito de controlar esse possível viés, razão pela qual limitaremos as informações específicas sobre os profissionais entrevistados e mesmo a descrição mais particularizada da Utip. Outro possível viés foi o fato de, na ocasião da pesquisa de campo, só haver mulheres contratadas para essa função nessa clínica. Assim, a ausência de homens entre os entrevistados impede a análise de eventuais diferenças entre os gêneros no processo de tomada de decisões.

As entrevistas foram realizadas no local de trabalho das profissionais, em horário escolhido por elas, fora do seu período regular de atividade. Foram realizadas 17 entrevistas de roteiro semiestruturado com as intensivistas pediátricas. Foi esclarecido às médicas que as perguntas se refeririam não apenas às decisões tomadas naquela unidade escolhida, mas também àquelas tomadas em qualquer Utip em que a entrevistada tenha exercido atividade profissional. As entrevistas ocorreram entre os meses de setembro e outubro de 2010 , após permissão para gravação de áudio e assinatura do termo de consentimento livre e esclarecido. A entrevista consistia em uma primeira parte, relativa aos dados de formação acadêmica e atividade profissional da profissional, seguida de questões abertas sobre a prática da LSV. A análise das entrevistas foi realizada segundo a técnica de análise de conteúdo desenvolvida por Bardin ${ }^{13}$.

No questionário elaborado para a pesquisa, havia um grupo de perguntas voltado para a identificação de situações em que se justificaria, do ponto de vista do entrevistado, a limitação do suporte de vida e um segundo grupo de perguntas que tentava identificar os valores nos quais as médicas se baseavam para a tomada de tal decisão. Após a leitura flutuante, em que foi realizado o primeiro contato 
com o texto, leituras repetidas permitiram o reconhecimento e a criação das categorias para análise e a identificação da matriz teórica da bioética nos argumentos apresentados para justificar ou não a suspensão do suporte vital dos pacientes.

\section{Resultados e discussão}

Foram entrevistadas 17 das 19 médicas que trabalhavam na unidade escolhida. Uma intensivista não foi entrevistada por estar de licença médica e a outra, conforme informado, por ser a pesquisadora principal do estudo. A faixa etária das entrevistadas variou de 30 a 55 anos, e a maior parte delas tinha mais de dez anos de formada. Nove médicas cursaram a faculdade de medicina em universidades públicas. Todas as entrevistadas têm a pediatria como especialização e nove possuíam pós-graduação em terapia intensiva pediátrica, incluindo todas aquelas com menos de dez anos de formadas. Seis entrevistadas cursaram ainda outra especialização. Treze médicas exerciam, no período da pesquisa, a função de intensivistas pediátricas em uma ou mais unidades além da escolhida para o estudo, e todas as entrevistadas já tinham tido a experiência de exercer tal função em Utip do setor público.

Todas as entrevistadas consideraram a limitação do suporte de vida tema difícil e delicado. Apesar de estudos mostrarem que mais da metade das mortes em Utip é precedida de alguma medida de LSV ${ }^{9}$, sete entrevistadas informaram nunca ter ouvido falar do termo. Quando solicitadas a definir o conceito, todas o fizeram como sinônimo de não reanimar e/ou não oferecer novas terapias. Embora a ordem de não oferta e a ordem de suspensão de tratamentos que não serão suficientes para recuperar o bem-estar de um paciente sejam moralmente equivalentes, nenhuma delas cogitou a possibilidade de suspensão de alguma terapia, mesmo quando questionadas diretamente sobre o fato. Onze das 17 entrevistadas admitiram já ter praticado a LSV, mas mais da metade apenas em situações pontuais. Das que negaram a prática, três conheciam o termo. A maioria das situações em que a LSV foi aventada referia-se a crianças em morte encefálica.

As normas para diagnóstico de morte encefálica foram publicadas em 1997 pelo Conselho Federal de Medicina e versam sobre a retirada do suporte vital daqueles pacientes com morte encefálica confirmada cuja família foi comunicada e que se acham impossibilitados de se tornarem doadores de órgão ${ }^{14}$. A retirada do suporte, nesse caso, tem inclusive apoio legal. Entretanto, o conceito de limitação do suporte de vida refere-se a processo de limitação de suporte naqueles pacientes ainda com vida e, dessa forma, não se aplica a pacientes em morte encefálica. Esse desconhecimento de conceitos, ou talvez o receio de alguma punição, acaba por gerar sofrimento nas famílias e gastos desnecessários para o responsável pelas despesas com a internação, seja a própria família do paciente, seja o Sistema Único de Saúde, seja o seguro-saúde.

Sendo a teoria dos quatro princípios ou principialismo de Beauchamp e Childress ${ }^{15}$ a corrente hegemônica da bioética no mundo, inclusive no que diz respeito às publicações de pesquisas internacionais, acreditávamos que as argumentações éticas utilizadas pelas entrevistadas, se presentes, se refeririam aos princípios dessa corrente. Todas as entrevistadas mencionaram expressões que refletiam mais de um princípio nas tomadas de decisões, mas apenas uma delas mencionou os quatro princípios, utilizando-os nominalmente. Essa médica frequenta reuniões de uma comissão de bioética em outro hospital. A menção a princípios como a não maleficência e beneficência parece estar relacionada com a tradição hipocrática, e não com o principialismo proposto por Beauchamp e Childress.

A não maleficência foi o princípio mais presente nos discursos das médicas, citado por 13 das 17 entrevistadas. Existe clara preocupação, por parte delas, com os procedimentos dolorosos impostos aos pacientes. Segundo artigos relacionados, esse deveria ser o princípio determinante para a escoIha de condutas terapêuticas nos casos de terminalidade ${ }^{16}$. Tal princípio, presente no pensamento hipocrático de primeiro não causar o mal (primun non noccere), parece ser a principal justificativa das intensivistas pediátricas participantes para a não oferta de tratamentos considerados fúteis para o paciente em estágio terminal, mas não parece forte o suficiente para legitimar a suspensão desses tratamentos. Todas as entrevistadas informaram que se fundamentavam no princípio da não maleficência em sua tomada de decisão, três das quais, paradoxalmente, admitiram já ter praticado medidas passíveis de ser reconhecidas como fúteis.

A beneficência, considerada pelos médicos a base moral de suas ações, foi mencionada em apenas 9 das 17 entrevistas. Muitos médicos, preocupados em "fazer o bem" a seus pacientes, interpretam essa expressão como a obrigação de utilizar todo o arsenal terapêutico disponível para todos os pacientes e acabam, de fato, provocando a distanásia naqueles considerados em estágio terminal. A maioria 
dos médicos que dizem fazer uso desse princípio utiliza simultaneamente a não maleficência como forma de pesar riscos e benefícios para determinar o tratamento a ser utilizado, atitude que está em conformidade com a tradição da ética médica, expressa em dois preceitos presentes desde o Juramento de Hipócrates: Aplicarei os regimes para o bem do doente segundo o meu poder e entendimento, nunca para causar dano ou mal a alguém ${ }^{17}$.

Estudo pediátrico sobre o fim de vida realizado no Brasil demonstrou que os princípios de beneficência e não maleficência foram utilizados em $83 \%$ dos casos contra apenas $50 \%$ de citações sobre a qualidade de vida ${ }^{16}$. Na presente pesquisa, 12 das 17 entrevistadas mencionaram a qualidade de vida como o argumento considerado no momento da decisão. Mas, dessas 12, apenas 2 médicas disseram que a qualidade de vida deve ser julgada pelo próprio indivíduo, o que é dificultado no caso de pacientes ainda sem autonomia plena como as crianças. Todas as outras 10 médicas disseram utilizar fatores objetivos para julgar qualidade de vida, como a capacidade de realizar determinadas tarefas e formas de interação com o meio, em uma tentativa de tornar mais imparcial sua avaliação.

A ideia partilhada pelas entrevistadas é que nem os pais nem qualquer representante legal estariam em condições de defender melhor os interesses maiores das crianças do que os próprios médicos, embora essa posição seja contrária à legislação brasileira, que torna obrigatória a participação da criança e do adolescente, em separado ou na companhia dos pais ou de seus representantes, em atos de medidas relacionadas com a promoção de seus direitos e proteção, devendo suas opiniões ser necessariamente consideradas ${ }^{18}$. O paternalismo implícito nessa posição é de fato injustificável se entendemos que o esforço de se colocar no lugar do outro será inútil, dada a impossibilidade de se compreender efetivamente o que o outro, com suas experiências de vida e valores próprios, considera como melhor para si e o que pode realmente ser melhor para ele.

O emprego desmedido da tecnologia, os recursos escassos e a falta de leitos de unidade de terapia intensiva levam muitos autores a utilizar o princípio da justiça como fundamentação para a tomada de decisão nos casos de LSV. Em 2004 Torreão observou, ao realizar uma pesquisa no Brasil sobre o manejo de pacientes em estágio terminal, que $72 \%$ dos médicos citaram o uso inapropriado de recursos como elemento que contribuiu para a decisão sobre a limitação do suporte de vida ${ }^{16}$. Segundo alguns pesquisadores, a justiça, mesmo sendo um princípio relevante, não deveria ser utilizada como fator determinante para essa decisão, porque, mais do que indicadores econômicos, a decisão sobre limitar a vida de um doente em estágio final engloba valores subjetivos do próprio paciente $3,3,16,19,20$.

Tal enfoque teórico parece vir ao encontro da perspectiva das entrevistadas, pois apenas 3 das 17 participantes desta pesquisa mencionaram ações que teriam como fundamento o princípio da justiça. Da mesma maneira que se observou nas pesquisas citadas, nenhuma de nossas entrevistadas considerou esse princípio como fator decisivo na tomada de decisão. Esse dado pode ser justificado por uma possível incompreensão sobre quando é pertinente se considerar o princípio da justiça no cuidado em saúde. Não tivemos informações suficientes para essa análise, sendo possível apenas formular uma hipótese explicativa.

Decisões referentes à LSV devem ser tomadas com avaliação individual de cada paciente em questão. Aspectos culturais, religiosos, benefícios e julgamentos da qualidade de vida são critérios subjetivos que exigem tolerância e reflexão por parte de todos aqueles envolvidos no processo ${ }^{21-24}$. Apenas quatro entrevistadas reconheceram que a decisão de LSV deve ser realizada caso a caso, não havendo situações nas quais tal decisão possa ser tomada sem exceção. Apesar da recomendação de que toda a equipe participe do processo, assim como o paciente e a família, apenas seis entrevistadas mencionaram que a LSV deve ser decidida com auxílio de equipe multidisciplinar. As onze médicas restantes disseram acreditar que outros profissionais além do médico e da família acabam por tumultuar o processo, tornando-o improdutivo.

Entretanto, ao contrário do encontrado nesta pesquisa, consideramos que a participação de outros profissionais no processo deliberativo garante uma decisão coesa que, quando não obtida, pode favorecer o surgimento de conflitos, dificultando a relação com a família e o plano terapêutico do paciente. Essa postura das entrevistadas ratifica a imagem onipotente que o médico tem de si, pouco tolerante com opiniões discordantes. O julgamento de valores morais, passo importante nesse processo, independe de grandes conhecimentos teóricos, mas exige respeito e tolerância ${ }^{25}$.

A participação da família no processo de LSV foi considerada importante por todas as médicas. No entanto, no caso de opiniões discordantes entre família e médico, apenas duas entrevistadas manteriam o tratamento oferecido e insistiriam no acordo. Para as demais, a decisão médica deve preponderar sobre a da família. 
A posição assimétrica entre o poder de decisão do médico e o da família que frequentemente transparece no discurso das entrevistadas, foi marcada por subterfúgios destinados a fazer que suas opiniões prevalecessem, como não serem explícitas quanto às propostas de tratamento, deixando de oferecer medidas terapêuticas que não acham, segundo seu ponto de vista, válidas. Soma-se a isso a interpretação própria que fazem da expressão "eu não quero que se retire nada", que muitos pais pronunciam como forma de se manifestar contra medidas de LSV e que as entrevistadas entendem não como uma proibição na ordem de retirada, mas como permissão para as ordens de não oferta e de não reanimar.

Algumas das razões citadas pelas entrevistadas para justificar essa atitude foram "problemas do lado familiar no binômio médico-família", os quais, segundo alegaram, se manifestavam na incapacidade intelectual dos pais de compreender o real estado dos filhos ou seu grande envolvimento emocional. O possível sentimento de culpa que os pais experimentariam por terem decidido pela morte dos filhos também é uma justificativa dada nas leis e normas de alguns países para legitimar o paternalismo médico ${ }^{21,26}$. Entretanto, pesquisas estadunidenses demonstram que $85 \%$ das famílias gostariam de ter a palavra final durante a LSV do seu familiar e que o processo de luto é mais bem elaborado naqueles pais que acompanharam o tratamento e participaram das discussões sobre limitação do suporte de vida ${ }^{27}$. A justificativa de incapacidade intelectual do familiar pode, na verdade, ocultar a baixa qualidade de comunicação entre médico e paciente-família e ser a razão do surgimento de conflitos durante a LSV.

A posição paternalista adotada pela maioria das entrevistadas ratifica o desconhecimento médico sobre o tema LSV e reforça a ideia de que os médicos se colocam como as pessoas mais capazes para realizar a escolha adequada, mesmo que tal escolha implique valores subjetivos de difícil julgamento. Modelos de decisões substitutas, como o do melhor interesse, criado por Beauchamp e Childress ${ }^{15}$, em que a atitude escolhida deve ter em vista a melhor opção para a criança, não conseguem responder a esse conflito na decisão por terceiros e descaracterizam, segundo o ponto de vista dos autores deste artigo, o conceito de autonomia.

Apenas uma entrevistada disse que o doente deveria participar do processo decisório, o que faz crer que as demais entrevistadas os consideram pessoas não autônomas, cujo poder legal de decisão está centrado na figura dos pais. Isso reforça o hábi- to que pediatras têm de não consultar as crianças, sempre que possível, sobre assuntos relacionados à sua saúde. A Declaração de Mônaco ${ }^{28}$, redigida no ano 2000 em simpósio internacional sobre bioética e direitos da criança, orienta que as crianças devem tomar parte na tomada de decisão sobre sua saúde, de maneira crescente conforme o desenvolvimento de sua autonomia e, em decorrência, os médicos deveriam sempre buscar o assentimento desses pequenos pacientes. No Brasil, o Estatuto da Criança e do Adolescente e o Código Civil também normatizam a participação do menor e de sua família no processo decisório ${ }^{18,29}$.

Muitas entrevistadas acreditam que a normatização das condutas diante de um paciente terminal poderia facilitar a argumentação na tomada de decisão sobre a LSV. Entretanto, a existência de protocolos em relação ao tema não é capaz de responder aos conflitos éticos desses casos. Como proceder para julgar o que é "melhor para o outro", o que é qualidade de vida ou a quem cabe a palavra final são questões cujas respostas não se encontram em protocolos. É necessário ponderar acerca dos valores, processo que não comporta critérios fixos e que exige o estudo caso a caso.

\section{Considerações finais}

Os argumentos que acreditávamos que seriam usados pelas entrevistadas não se confirmaram. As intensivistas pediátricas entrevistadas nesta pesquisa, apesar de utilizarem expressões que remetem aos princípios da corrente principialista, não pareceram estar familiarizadas com os fundamentos dessa teoria. Em suas respostas, demonstraram grande preocupação em não desrespeitar o que supõem ser a interdição legal da limitação de suporte de vida, embora o façam em suas práticas. Em contrapartida, declararam desconsiderar os preceitos legais que reconhecem ter os pais e os menores, no limite de suas competências, o direito de decidir sobre fatos e acontecimentos de suas vidas. Questionando desde o equilíbrio emocional dos pais até seu desconhecimento técnico, elas parecem reivindicar para si a autoridade técnica e uma consequente autoridade moral para decidir em nome de seus pacientes até mesmo quando devem morrer, sem considerar imprescindível que os demais interessados participem da decisão.

O paternalismo expresso nas entrevistas tem suas raízes na própria formação do Estado brasileiro e na fragilidade democrática de nosso país, que apenas a partir da promulgação da Constituição de 1988 pas- 
sou a contar com legislação que protege os direitos individuais e coletivos dos cidadãos. Embora a legislação já esteja disponível, ainda levará um tempo até que esses direitos sejam efetivamente assegurados a todos. Tais dificuldades também se manifestam, como visto, nas relações profissionais entre médicos e pacientes. Também nesse campo se fazem necessárias ações específicas, no caso, de cunho educativo, para que as discussões sobre a morte, o morrer, a distanásia e a autonomia dos pacientes não sejam tão superficiais como aqui foi demonstrado. A bioética pode ser um instrumento importante nesse processo, ao permitir maior investimento nas discussões sobre os aspectos morais da prática profissional, buscando a formação de profissionais comprometidos com o diálogo e o respeito aos direitos humanos.

Trabalho realizado no âmbito do Programa de Pós-graduação em Bioética, Ética Aplicada e Saúde Coletiva da Fiocruz/UFRJ/UFF/UERJ.

\section{Referências}

1. Ariés P. História da morte no Ocidente. Rio de Janeiro: Nova Fronteira; 2012. p. 86

2. Ristagno G, Weil MH. History of critical care Medicine: the past, the present and the future. In: Gullo A, Besso J, Lumb PD, Willians GF, editors. Intensive and critical care medicine. [Internet]. Milan: World Federation of Societies of Intensive and Critical Care Medicine; 2009 [acesso 10 abr 2013]. Disponível: file:///C:/Users/meliane/Downloads/0912f50b491b024f66000000.pdf

3. Rego S, Palácios M. A finitude humana e a saúde pública. Cad Saúde Pública. 2006;22(8):1.75560.

4. Luce JM, White DB. A history of ethics and law in the intensive care unit. Crit Care Clin. 2009;25(1):221-37.

5. Siqueira-Batista R. A ressurreição de Frankenstein: uma metáfora das unidades de terapia intensiva contemporâneas. In: Schramm FR, Rego S, Braz M, Palácios M, organizadores. Bioética: riscos e proteção. Rio de Janeiro: Editora UFRJ; 2005. p. 148-63.

6. Othero JCB. Atitudes médicas nas últimas 24 horas de vida de pacientes adultos internados em três unidades de tratamento intensivo no Sul do Brasil [dissertação]. Porto Alegre: Pontifícia Universidade Católica do Rio Grande do Sul/Faculdade de Medicina de Porto Alegre; 2008.

7. Garros D. Uma "boa" morte em UTI pediátrica: é isso possível? J Pediatr. 2003;79(Supl. 2):S24354.

8. Levin PD, Sprung CL. Cultural differences at the end of life. Crit Care Med. 2003;31(5):S354-7.

9. Keenan HT, Dekema DS, O'Rourke PP, Cummings P, Woodrum DE. Attitudes toward limitation of support in a pediatric intensive care unit. Crit Care Med. 2000;28(5):1.590-4.

10. Rego S. Contribuições da bioética para a saúde pública. Cad Saúde Pública. [Internet]. 2007 [acesso 1ㅇabr 2013];23(11):2.530. Disponível: http://www.scielo.br/pdf/csp/v23n11/00.pdf

11. Diniz D. Quando a morte é um ato de cuidado: obstinação terapêutica em crianças. Cad Saúde Pública. [Internet]. 2006 [acesso 19 abr 2013];22(8):1.741-8, p. 1.742. Disponível: http://www. scielo.br/scielo.php?script=sci_arttext\&pid=S0102-311X2006000800023\&lng=en

12. Barbosa AP. Terapia intensiva neonatal e pediátrica no Rio de Janeiro: distribuição de leitos e análise de equidade. Rev Assoc Med Bras. 2002;48(4):303-11.

13. Bardin L. Análise de conteúdo. Lisboa: Edições 70; 2009.

14. Conselho Federal de Medicina. Resolução no 1.480, de 8 de agosto de 1997. A morte encefálica será caracterizada através da realização de exames clínicos e complementares durante intervalos de tempo variáveis, próprios para determinadas faixas etárias. Diário Oficial da União. 21 ago 1997;(160):Seção I, p. 18.227-8.

15. Beauchamp TL, Childress JF. Princípios de ética biomédica. São Paulo: Loyola; 2002.

16. Torreão LA, Pereira CR, Troster E. Ethical aspects in the management of the terminally ill patient in the pediatric intensive care unit. Rev Hosp Clin Fac Med São Paulo. 2004;59(1):3-9.

17. Juramento de Hipócrates. [Internet]. São Paulo: Conselho Regional de Medicina do Estado de São Paulo; [acesso 16 jul 2012]. Disponível: http://www.cremesp.org.br/?siteAcao=Historia\&esc=3

18. Brasil. Lei no 8.069, de 13 de julho de 1990. Dispõe sobre o Estatuto da Criança e do Adolescente e dá outras providências. [Internet]. Diário Oficial da União. 16 jul 1990 [acesso nov 2014]. Disponível: http://www. planalto.gov.br/ccivil03/leis/L8069.htm

19. Siqueira JE. A evolução científica e tecnológica, o aumento dos custos em saúde e a questão da universalidade do acesso. Bioética. 1997;5(1):41-8.

20. Levy MM, Mc Bride D. End-of-life care in the intensive care unit: state of the art in 2006. Crit Care Med. 2006;34(11):S306-8.

21. Lago PM, Devictor D, Piva JP, Bergounioux J. End-of-life-care in children: the brazilian and the international perspectives. J Pediatr. 2007;83(2):S109-16.

22. Charatan F. AMA issues guidelines on end of life care. BMJ. 1999;318(7.185):690. 
23. Australia. NSW Department of Health. Guidelines for end-of-life care and decision-making. [Internet]. Sydney: NSW Department of Health; 2005 [acesso 15 jan 2011]. Disponível: http:// www.health.nsw.gov.au/policies/gl/2005/pdf/GL2005_057.pdf

24. Moritz RD, Lago PM, Deicas A, Nilson C, Machado FO, Othero J et al. 1o Fórum do Grupo de Estudos do Fim da Vida do Cone Sul: proposta para atendimento do paciente portador de doença terminal internado em UTI. Rev Bras Ter Intensiva. 2009;21(3):306-9.

25. Möller LL. Pluralismo e tolerância: valores para a bioética. Rev HCPA. 2008;28(2):101-9.

26. Devictor D. Parents' autonomy versus doctors' paternalism: a rearguard battle [letter]. Pediatr Crit Care Med. 2007;8(4):400-1.

27. Lago PM, Garros D, Piva JP. Participação da família no processo decisório de limitação de suporte de vida: paternalismo, beneficência e omissão. RTBI. 2007;19(3):364-8.

28. Organização das Nações Unidas para a Educação, a Ciência e a Cultura. Associação Mundial dos Amigos da Criança (Amade). Declaração de Mônaco. Simpósio Internacional sobre a Bioética e os Direitos da Criança; 28-30 abr 2000; Mônaco. [Internet]. [acesso 5 ago. 2012]. Disponível: http:// ww2.prefeitura.sp.gov.br//arquivos/secretarias/saude/cepsms/0007/Biotica_Direitos_Criancas. pdf

29. Brasil. Lei no 10.406, de 10 de janeiro de 2002. Institui o Código Civil. Diário Oficial da União. 11 jan 2002 [acesso 5 ago 2012]. Disponível: http://www.planalto.gov.br/ccivil_03/leis/2002/ L10406compilada.htm

\section{Participação dos autores}

Grace Kelly Paranhos - concepção e delineamento do estudo, revisão bibliográfica, realização do trabalho de campo, análise dos dados, redação do artigo. Sergio Rego - concepção e delineamento do estudo, análise dos dados, redação do artigo e revisão crítica.

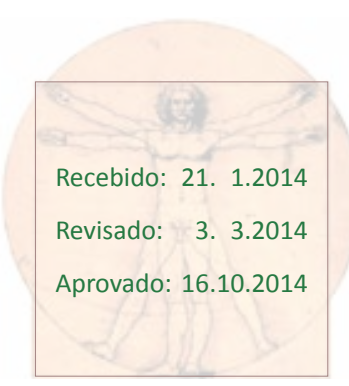




\section{Anexo}

Roteiro da entrevista

- Data de nascimento:

- Sexo:

- Ano de formatura da graduação médica? Faculdade:

- Especialização em pediatria?

( ) Sim

( ) Não

( ) Residência

( ) Especialização

( ) Outro. Qual?

- Formação em terapia intensiva pediátrica?

( ) Sim

( ) Não

( ) Residência

( ) Especialização

( ) Outro. Qual?

- Formação em outra especialidade?

( ) Sim. Qual?

( ) Não

- Titulo de especialista em terapia intensiva pediátrica?

( ) Sim. Ano:

( ) Não

- Titulo de especialista em outra especialidade?

( ) Sim. Qual?

( ) Não

- Em quantas unidades de terapia intensiva pediátrica você trabalha? Quais as características destas unidades? 1 a

2aㅡ

3 a

( ) Pública

( ) Pública

( ) Pública

( ) Privada

( ) Privada

( ) Privada

( ) Pediátrica

( ) Pediátrica

( ) Pediátrica

( ) Neonatal

( ) Neonatal

( ) Neonatal

( ) Mista

( ) Mista

( ) Mista
- O que você considera limitação do suporte de vida?

- Você já limitou o suporte de vida de alguma criança? Por quê? Em que situação?

- Já aconteceu de se limitar o suporte de vida de algum paciente pediátrico em um serviço que você trabalha? Qual era a situação?

- Essas decisões foram registradas no prontuário? Por quê ? Em que situações você acha que estas decisões devem ser registradas no prontuário?

- Você acha que existe alguma situação em que se justifica o limite do suporte de vida? Qual(is)? Por quê? Por que em algumas situações você acha que é razoável o limite do suporte de vida e em outras não? O que faz a diferença entre essas situações de modo a tornar em algumas situações isso aceitável e em outras não? Por que você acha que nunca é aceitável/justificável? Por que você acha que é sempre aceitável/justificável optar pela limitação do suporte de vida?

- Quem você acha que deve participar destas decisões? Qual deve ser o papel da equipe médica nestas decisões? E da família? E do paciente?

- Em sua opinião, que valores devem orientar a tomada de decisão nestes tipos de situação? Por quê? 
- Você acha, no que diz respeito ao limite de suporte de vida, que as suas decisões são diferentes quando você está trabalhando em serviços públicos ou privados? E a de outros médicos?

- Durante a sua graduação de medicina, você teve oportunidade de discutir sobre dilemas éticos?

( ) Sim

( ) Não
E sobre limite de suporte de vida?

( ) Sim

( ) Não

E na sua formação em pediatria?

( ) Sim

( ) Não

E na sua formação em terapia intensiva pediátrica?

( ) Sim

( ) Não 